\title{
An inverse problem in modelling of a symmetric gene network regulated by negative feedbacks
}

\author{
V. Golubyatnikov ${ }^{1,2 *}$, V. Gradov ${ }^{2}$ \\ ${ }^{1}$ Sobolev Institute of Mathematics SB RAS, Novosibirsk, Russia \\ ${ }^{2}$ Novosibirsk State University, Novosibirsk, Russia \\ *e-mail: vladimir.golubyatnikov1@fulbrightmail.org
}

Key words: Negative feedbacks, piece-wise linear dynamical systems, inverse problems

Motivation and Aim: We study one piece-wise linear dynamical system which describes functioning of a gene network regulated by negative feedbacks in order to find conditions of existence and uniqueness of periodic regimes of its functioning and show existence and uniqueness of solution of an inverse problem of identification of parameters of this system. Methods and Algorithms: The approaches to modelling of similar gene networks, description of phase portraits of corresponding dynamical systems and detection of their periodic trajectories (cycles) are presented in $[1,2]$. For some other non-linear dynamical systems, similar constructions were described in [3].

Results: For positive parameters $A, m, \alpha$, where $A>\alpha$, we consider symmetric piece-wise linear 3D dynamical system

$\frac{d x}{d t}=L(z)-m x ; \frac{d y}{d t}=L(x)-m y ; \frac{d z}{d t}=L(y)-m z$.

Here $L:[0, \infty) \rightarrow[0, \infty)$ is monotonic step-functions which corresponds in gene network to negative feedback, $L([0, \alpha))=A, L([\alpha, \infty))=0$. We show that the cube $Q=[0, A] \times$ $[0, A] \times[0, A]$ is invariant and decompose it to 8 blocks by hyperplanes $x=\alpha ; y=\alpha ; z=\alpha$. Note, that the system (1) is symmetric with respect to cyclic permutation of the variables $x \rightarrow y \rightarrow z \rightarrow x$.

Theorem 1. For the system (1), there exists unique piece-wise linear cycle $\boldsymbol{C}$ symmetric with respect to that cyclic permutation. This cycle $C$ travels through six blocks of the decomposition of the invariant domain $Q$.

Let $\tau$ be the period of this cycle $C$ which can be measured in experiments, and let the parameters $A$ and $m$ be known as well. Also, we assume that we can measure the time $t_{1}$ between two consecutive peacks of the graphs of the piece-wise linear functions $x(t), y(t)$, $z(t)$. At the same time these three functions are not assumed to be known.

Theorem 2. Let the parameter $A$ and the times $\tau, t_{1}$ for the system (1) be known, and $\alpha \in(0, A)$ be unknown. Then the inverse problem of determination of the parameter $\alpha$ has unique solution.

Conclusion: The main reason of our studied is the fact that the time measurements $\tau$ and $t_{1}$ of the oscillations in the gene network can be realized in non-invasive way. Similar inverse problem can be formulated for asymmetric dynamical systems of other dimensions as well. Acknowledgements: Supported by RFBR, (18-01-00057) and by complex program of basic research of SB RAS (0314-2018-0011).

\section{References:}

1. Ayupova N.B., Golubyatnikov V.P. (2014) On the uniqueness of a cycle in an asymmetric three-dimensional model of molecular repressilator. Journal of Applied and Industrial Mathematics. 8(2):1-6.

2. Golubyatnikov V.P., Kalenykh A.E. (2016) On structure of Phase Portraits of Some Nonlinear Dynamical systems. Journal of Mathematical Sciences. 215(4):475-483.

3. Glass L., Pasternack J.S. (1978) Stable oscillations in mathematical models of biological control systems. Journal of Math. Biology. 6:207-223. 